\section{Looking Ahead: Addressing Ethical Challenges in Public Health Practice}

\author{
Nancy M. Baum, Sarah E. \\ Gollust, Susan D. Goold, and \\ Peter D. Jacobson
}

\section{Introduction}

In recent years, scholars have begun to lay the groundwork to justify a distinct application of ethics to the field of public health. They have highlighted important features that differentiate public health ethics from bioethics, especially public health's emphasis on population health rather than issues of individual health. Articulations of public health ethics also tend to emphasize the role of social justice compared to the predominance of autonomy in the bioethical literature. ${ }^{1}$ Now that the field of public health ethics is developing a unique focus and a language of its own, including a code of ethics disseminated by the American Public Health Association, the future of public health ethics may well be global health ethics, focusing on issues of global justice. ${ }^{2}$ As public health ethics evolves from its nascent stage of reflection to a place of action and application in the national and global arenas, two interrelated developments will need to occur: (1) public health professionals, including practitioners, policymakers, and scholars, will need a richer understanding of the ethical challenges practitioners face on a daily basis and (2) scholars will need to develop useful tools (i.e., frameworks) that practitioners may employ for identifying and tackling these ethical challenges.

Based on our ongoing empirical work interviewing public health professionals ${ }^{3}$ and our scholarly activities in public health, we developed a framework to address the varied types of ethical challenges that policymakers and practitioners face in their work. We expect that this framework can be refined and advanced through empirical evaluation. As other scholars of public health ethics have pointed out, incorporating ethical reflection concurrently into public health practice and decision-making can create a more just, inclusive, and effective public health practice. ${ }^{4}$ Yet our

Nancy M. Baum, M.H.S., is a doctoral student in the Department of Health Management and Policy at the University of Michigan School of Public Health in Ann Arbor. Sarah E. Gollust is a Ph.D. candidate in the Department of Health Management and Policy at the University of Michigan School of Public Health in Ann Arbor. Susan D. Goold, M.D., M.H.S.A., M.A., is an Associate Professor of Internal Medicine and Health Management and Policy and the Director of the Bioethics Program at the University of Michigan in Ann Arbor. Goold's research focuses on the allocation of scarce health care resources and ethical aspects of health care financing and delivery using conceptual and empirical models. Peter D. Jacobson, J.D., M.P.H., is a Professor of Health Law and Policy at the University of Michigan School of Public Health in the Department of Health Management and Policy, where he is also the Director of the Center for Law, Ethics, and Health. Jacobson's research focuses on examining the role of the courts in shaping health care and public health policy. 
investigation of ethical issues faced by practitioners led us to the conclusion that a more prospective (even preventive) approach could be useful for practitioners in their daily work.

In this paper, we offer an analytic framework designed to assist policymakers and practitioners in managing the public health ethical tensions they now confront. Unlike other commentators on public health ethics, our level of analysis is not aimed at articulating theoretical ethical principles that underlie public health. ${ }^{5}$ Rather, our analytical task is grounded within daily public health practice, particularly at the level of local governmental public health departments. While it is based on our U.S. research experiences, we believe our framework could have global applicability. Our goal is to provide a contribution to public health ethics which is both responsive to public health practice and analytically rigorous.

\section{Emergence of Public Health Ethics}

Historically, the field of bioethics has largely focused on individuals - most prominently professional ethics for physicians and nurses - in the context of medi- cal treatment and research. Bioethical considerations have been oriented toward informed consent on treatment and research, reproductive and end-of-life decision-making, and applications of emerging technologies in personal health care. While philosophers have not explicitly ranked autonomy highest among ethical principles, many commentators have noted that the principal construct addressed in bioethical inquiry is respect for persons, particularly respect for patient autonomy. ${ }^{6}$ Over time, autonomy has become, at a minimum, primus inter pares, and some have argued that autonomy now supersedes other bioethical principles. ${ }^{7}$ Other conceptual frameworks for bioethics, including narrative approaches, feminist theories (especially those focused on caring), and casuistry, still focus primarily on individuals or interpersonal relationships. Even commentary on access to health care often focuses on individual rights, and nearly always speaks about health care and not health.

The emphasis on health as a common good (i.e., population health concerns) distinguishes public health from individually oriented health care. Communally shared health goals, such as herd immunity

Figure |

Traditional Issues and Foundational Principles and

Values in Bioethics and Public Health Ethics

TRADITIONAL ISSUES

\begin{tabular}{|c|c|c|}
\hline BIOETHICS & $\begin{array}{l}\text { - Informed consent and patient agency } \\
\text { - Reproductive and end-of-life } \\
\text { decision-making } \\
\text { - Use of emerging technologies/ } \\
\text { bedside rationing } \\
\text { - Clinical research ethics } \\
\text { - Confidentiality }\end{array}$ & $\begin{array}{l}\text { Autonomy } \\
\text { Beneficence } \\
\text { Non-maleficence } \\
\text { Justice/fairness } \\
\text { Utility } \\
\text { Caring }\end{array}$ \\
\hline $\begin{array}{r}\text { PUBLIC HEALTH } \\
\text { ETHICS }\end{array}$ & $\begin{array}{l}\text { - Vulnerable populations/uninsured } \\
\text { - Infectious disease control } \\
\text { - Social determinants of health } \\
\text { - Cost-effective decision-making } \\
\text { - Emergency preparedness }\end{array}$ & $\begin{array}{l}\text { Population-level utility } \\
\text { Evidence } \\
\text { Justice/fairness } \\
\text { Accountability } \\
\text { Costs/efficiencies } \\
\text { Political feasibility } \\
\text { Beneficence } \\
\text { Non-maleficence } \\
\text { Autonomy }\end{array}$ \\
\hline
\end{tabular}

Note: The above table depicts differences between bioethics and public health ethics, both in terms of the issues relevant to each field and the principles and values that underlie decision-making in each. Autonomy has been characterized as a central principle in bioethics inquiry, and thus appears in bold in the figure. In contrast, in our conception of public health ethics, we do not emphasize one particular principle; instead, we have highlighted five considerations we believe to be most relevant to decision-makers in public health. 
gained through mass vaccination, clean water, or protection from bioterrorist threats, are more than simply the aggregation of individual health goals: they are goods held in common. The inadequacy of an autonomy-focused approach to communitylevel public health issues has led many commentators to suggest that public health ethics is a field of inquiry in its own right. ${ }^{8}$ Some argue that bioethics, properly construed, can include public health, environmental health, and other population-centered domains. ${ }^{9}$ In contrast, we contend that public health decisions may benefit from systematic deliberation of certain ethical considerations tailored to a public health approach. Figure 1 illustrates our interpretation of the different applications of ethics in medical care and public health.

\section{Frameworks for Public Health Ethics}

Scholars have introduced several broad, systematic approaches to understanding and evaluating public health ethics. ${ }^{10}$ Between 2001 and 2003, three published frameworks were directed toward public health professionals, each with a slightly different focus. ${ }^{11}$

Nancy Kass developed an analytic tool to consider ethical implications in program planning and policy. ${ }^{12}$ Her framework highlights the importance of considering the goals of a program, its effectiveness, known or potential burdens, minimizing burdens, fair implementation, and a fair balance of benefits and burdens. James Childress and colleagues, in addition to outlining the multiple moral considerations inherent in public health, described five conditions that must be met in order to justify actions that override important moral considerations (such as individual liberty). ${ }^{13}$ These are the program or policy's effectiveness, proportionality of benefits and burdens, necessity, least restrictive infringement, and public justification. Phillip Nieburg, Ruth Bernheim, and Richard Bonnie described four steps for practitioners to evaluate the ethical issues embedded in daily practice decisions. ${ }^{14}$ These include assessing the problem from the perspectives of the relevant stakeholders, locating the ethical tensions within the problem, considering various solutions, and evaluating the process to identify methods for improving current and future practice.

Broadly speaking, these frameworks have in common their intent to assess goals, effectiveness, and benefits and burdens of public health interventions or actions. They consider the distributional consequences of interventions, and assess fairness and the role of justice in public health actions. The frameworks enable consistency among decisions, help ensure that decisions reflect stakeholder and/or public values, and illuminate the ethical tradeoffs and balancing of moral
Setting priorities for allocating finite resources blic health e major challenges made even more difficult as budgets decline. These and multiple
other ethical issues require practitioners and policymakers to negotiate among competing priorities in their communities and to apply normative judgments when resolving conflicts.

considerations that underlie public health practice. Frameworks enhance practitioners' or scholars' ability to consider ethical issues in public health in a structured and formal way.

At least two other distinct frameworks have had a significant role in laying the groundwork for ethical analysis in public health. In 2006, Lawrence Gostin and Madison Powers proposed an approach to public health ethics that focuses explicitly on vulnerable populations, both domestically and globally, the fair distribution of resources and services, social determinants of health, and adequate and stable funding for public health systems..$^{15}$ They place particular emphasis on the principle of justice. In contrast, the analytic framework of Norman Daniels and James Sabin emphasizes procedural justice. ${ }^{16}$ Rather than emphasizing a particular ethical principle to guide decisionmaking, they instead suggest instituting a fair process by which decisions should be reached and evaluated. Recognizing the inherently political nature of public health actions and that decision-makers do not always agree on which action-guiding principles should apply, Daniels and Sabin suggested that in lieu of consensus, public health professionals should assess whether the processes for ethical decision-making are fair. Their approach, which they called "accountability for reasonableness," identifies four necessary conditions for ensuring justice and legitimacy in decision-making: publicity, relevance, appeals, and enforcement. The process must incorporate a publicly available rationale for decisions, a mechanism for dispute resolution, and a regulatory element to enforce decisions. The relevance condition requires that the reasoning behind actions is consistent with that of fair-minded people and is relevant to all involved. 
Finally, some additional efforts to formally assess ethical issues embedded in public health practice have emerged in recent years during widespread public concern over bioterrorism and other emergent disease threats. These frameworks target the balancing of individual liberty and liberty-infringing public health authority, such as decisions to invoke quarantine or isolation in response to pandemic flu. ${ }^{17}$ Practitioners, of course, only rarely face such an extreme situation of balancing public health authority with liberty, as when confronted with emergency situations or recalcitrant patients infected with some transmissible disease. Other infringements on individual liberty, however, such as mandatory vaccinations, confront practitioners more often, and are just one example of the many areas of ethical tension that emerge in daily public health practice. As we discuss below, our framework recognizes that public health practice fundamentally requires practitioners to balance various considerations rather than follow any one organizing principle.

\section{Public Health Ethics in Practice}

In considering the potential value and utility of ethical clarification and the use of frameworks to approach ethics in public health practice, we rely heavily on the experiences and insights of local public health practitioners related during in-depth interviews, as well as our own teaching and research in public health law, public health ethics, and resource allocation. No one disputes that public health practice is suffused with morally challenging issues at the macro level (resource allocation) and the micro level (day-to-day decisions). For instance, environmental health workers must manage the conflicting priorities of individual property owners and community standards for sanitation, a role in which they often experience political and community pressure. Setting priorities for allocating finite resources and assessing the value of public health services are major challenges made even more difficult as budgets decline. These and multiple other ethical issues require practitioners and policymakers to negotiate among competing priorities in their communities and to apply normative judgments when resolving conflicts.

We recognize that public health practitioners may lack the experience, time, resources (including training), or even motivation to deliberately consider ethics in their daily work. Indeed, in a number of our interviews, practitioners suggested that relying on professional experience or one's own personal moral foundation is often sufficient in negotiating challenging ethical issues. However, we believe that greater clarification of the ethical underpinnings of their decisions can add value to public health work for several reasons. First, ethical clarification ensures that officials and practitioners unmask normative assumptions and explicitly, rather than implicitly, analyze values during their assessments. Given that most public health activities in the United States are conducted by state and local governmental agencies and rely upon public funds, ${ }^{18}$ accountability to the public requires transparency. Transparency also engenders trust in communities, ultimately rendering public programs more effective. ${ }^{19}$

Second, ethical clarification helps balance economic analysis. Cost-effectiveness analysis and other forms of economic scrutiny can be important elements in the decision-making process, but if they are the only criteria used, they could lead to unsatisfactory health decisions. Many public health services are valued beyond their economic utility. For instance, whether or not preventive services prove to be cost saving, they may nonetheless be desirable because they significantly improve individual and community health and lifestyle. ${ }^{20}$ Emergency preparedness activities may be highly valued by the community, despite their great expense, for the increased sense of security that comes from the knowledge that their public health department has an emergency plan and staff trained to execute a plan when necessary. Government agencies may justify support for programs that are not shown to be cost saving by articulating the values, such as caring for the least well off, that support the governmental action. Ethical clarification can provide the structure in which to do so.

Third, clarifying the ethical considerations integrated into a particular policy or program decision can help to illuminate decision-makers' conceptions of the appropriate scope of public health practice. An ongoing debate exists in the field about whether public health ought to deal primarily with population protection, such as preventing the spread of communicable diseases, or whether it should serve as a safety net for the most vulnerable, and how to balance those competing missions. ${ }^{21}$ Practitioners' values influence their decisions about which programs a health department runs, assuming they have latitude in making such decisions. Ethical clarification helps practitioners identify the explicit ethical tradeoffs at stake, encourages consultation with others, and provides a framework for justifying the decision.

Similarly, the boundaries of public health practice are unclear. Currently, a robust scholarly debate exists about the desirability of allocating scarce public health resources to issues such as chronic disease or obesity that are not based on the traditional public health model of preventing disease transmission 
to the population. As an example, some argue that issues such as teen violence or the social determinants of health should not be under the purview of local governmental health agencies in the U.S. ${ }^{22}$ In certain communities, these issues may be excluded from the realm of public health and relegated to other arenas, such as law enforcement or education. Other communities may view youth violence as a significant threat to the public's health and may dedicate public health resources to combat it. Ethical clarification when considering or designing programs and policies can help practitioners better define their priorities and their boundaries, especially when they run into the controversial margins of public health practice. ${ }^{23}$

These justifications - transparency, balancing cost considerations, and articulating the scope and mission of public health - support the desirability of a contextbased and systematic evaluation of ethical issues in practice. Our ongoing empirical work suggests that practitioners will encounter ethical issues during (at least) two different points in time. One occurs during the political process of allocating resources; the other occurs in day-to-day practice. The former, if anticipated and dealt with prospectively, might mitigate challenges "downstream" in public health practice. For example, practitioners may see that a particular health problem in their community is endemic, such as lead poisoning, but the health department lacks the funds or authority to perform environmental investigations or rehabilitation. Conversely, practitioners may be required to devote their time and energy to a program - perhaps because of restricted funds or political influence - that their experience suggests is not a top health priority for their community. Clearly, these types of ethical issues must be addressed upstream during funding and programmatic decisions. By the time practitioners confront these types of challenges in daily practice, the range of feasible, acceptable, and ethical actions available may be quite narrow.

Other day-to-day ethical tensions must be handled within the specifics of public health interventions and enforcement considerations, instead of prospectively. Frequently, these tensions will also involve legal and political components that are not easily disentangled. Though ethical, legal, and political issues do not always co-occur, and each has distinct features, there often exists sufficient overlap that in many cases these three types of issues must be considered together, instead of attempting to impose artificial distinctions. Indeed, we believe that a dominant characteristic of public health ethics, and one that complicates how practitioners respond to ethical challenges, is the nearly constant intermingling of the ethical, political, and legal.

\section{A Framework to Manage Ethical Challenges in Practice}

We have two chief concerns about the existing frameworks for ethical analysis applied to public health. First, in general, these frameworks endeavor to identify and resolve moral conflicts in public health, but they do not adequately emphasize the potential for avoiding the creation of ethical tensions. In our view, decision-makers should have a framework that can be applied prospectively during the conceptualization and planning stages of public health actions to limit, as much as possible, the inadvertent creation of ethical challenges for practitioners in the field.

Second, the field of public health is complex and multidisciplinary, and therefore any guiding framework must reflect that complexity. Practitioners come from a variety of backgrounds and training, and are likely to hold a host of values that inform their views of ethical public health practice. A public health practitioner trained in epidemiology, for instance, may place a high value on evidence-based decisionmaking, while one trained in economics may emphasize utility, and another trained in environmental health may underscore the needs of future generations. If a framework focuses narrowly on one particular perspective, other types of practitioners may not find it useful. Though the ideal of social justice, for instance, can play a critical function in developing best practices in public health, if we attend only to justice in our analyses, then we may overlook vital issues that are not naturally illuminated by that single ethical precept. For example, planning a needleexchange program intended to reduce transmission of blood-borne illnesses, if analyzed strictly from the point of view of social justice, correctly identifies the needs of at-risk, vulnerable populations. However, a focus on justice alone fails to take into account whether implementation is politically feasible or acceptable by a particular community. Nor does it highlight the normative tradition in public health of identifying and using high-quality evidence to guide decision-making.

Together, the six considerations of our framework (as described below and highlighted in bold in Figure 1) create a structured guide that decision-makers may find helpful, both to identify potential ethical issues in public health practice and to possibly reduce the creation of ethical tensions.

\section{Determine Population-Level Utility of the Proposed Action}

Any proposed public health action should be useful to a community (in the present or predictably in the future) and should advance the well-being of those 
affected. The criterion of utility presupposes that decision-makers have a clear and accurate understanding of the expectations and values of members of the community in order to assess program utility. If programs are not consistent with community need, then public resources would be used ineffectively and unmet need would persist.

\section{Demonstrate Evidence of Need and Effectiveness of Actions}

Prior to designing and implementing public health programs, policymakers have an obligation to identify and to use the best available evidence to determine need for the proposed program and to support its effectiveness. Identifying the scientific basis for public health practice is appropriately becoming a foundational principle in the field of public health. Since building evidence takes time and resources, we recognize that there will be situations in which, for instance, relevant epidemiological studies are not yet available or program evaluation has not taken place (e.g., particularly where resources are severely strained as in resource-poor countries). Where evidence $i s$ available, decision-makers should seek it out and use it to justify program implementation. Used prospectively, this epistemic condition would also recommend that policymakers build needs-assessment and evaluation processes into their public health budgets.

\section{Establish Fairness of Goals and Proposed Implementation Strategies}

Decision-makers should be clear about what they intend to accomplish through proposed actions and assess whether the expected benefits and the burdens of the actions are likely to be distributed equitably in the community. There should be sufficient justification for unequal distributions of benefits and burdens. Evidence that vulnerable populations (those who have endured disproportionate illness burdens or those with the greatest need) will be afforded greater benefits or fewer burdens can justify unequal, but equitable, distribution. Similarly, one might justify providing benefits to one subgroup of a community by appealing to the need to gather evidence of effectiveness prior to widespread implementation and investment. Explicit balancing of the competing values of fairness and evidence might help practitioners identify alternative options (i.e., a fair process for selecting the initial subgroup) and hence respect both justice and the need for evidence.

\section{Demonstrate Accountability}

Given the prominent role of government in public health, practitioners and policymakers inherently face the need to be accountable to the public, including the community they serve. Transparency - making public and explicit one's assumptions, justifications, and reasoning - contributes to accountability and is essential for establishing trust with the public. Credible, scientifically sound decision-making further enhances accountability, as does responsible and competent stewardship of public funds.

\section{Assess Expected Efficiencies and Costs Associated with the Proposed Action}

Although it may appear as an anathema to some that ethical analysis should include cost considerations, we see issues of cost, utility, and fairness as deeply interdependent, requiring concurrent analyses. Costs represent an important component of the burden associated with the implementation and maintenance of public health programs. Where resources are limited, costs associated with one program represent opportunities forgone for alternative programs. Without systematically examining the expected costs of a proposed program, decision-makers cannot fully understand the tradeoffs involved in implementing such an initiative and therefore cannot perform a thorough assessment of its ethical dimensions. Too often, costs are viewed as an impediment to better (or more ethical or more just) action, whereas prudent stewardship of limited resources should be viewed as a morally admirable quality of public health practitioners.

\section{Consider Political Feasibility and Community Acceptance}

Local public health in the United States is inherently a political endeavor. As Fitzhugh Mullan synthesized, "Public health practitioners...do not [work] in a laboratory but in the public arena - a domain governed by political forces and politicians whose agendas are larger and more variable than those of the public health worker."24 The public health agenda depends largely on funding streams at the federal, state, and local levels, as well as the interests of local county executives, commissioners, and mayors. Public health programs promulgated without considering their potential for political feasibility and community acceptance will not go far toward improving public health - an imperative of ethical public health practice. Incorporating political feasibility into decisions does not, however, mean limiting options to popular ones. In fact, ethical decision-making that is unpopular demonstrates moral courage. For example, fluoridation of public water supplies is unpopular in some areas because it is viewed as a constraint on individual liberty, yet it is pursued by public health officials because of the known dental benefits. ${ }^{25}$ 
Finally, several other principles that have historically been central in the development of the field of bioethics - autonomy, non-maleficence, and beneficence - have a role in public health, but may be relevant to a lesser degree than they have been in bioethics; thus, they appear in the lower right-hand box in Figure 1, albeit below the six balancing considerations we emphasize here. Certainly the principle of autonomy in particular has had an important place in understanding the appropriate limits of public health actions. For instance, public health officials balance their authority to impose quarantine or to compel done. Incorporating the more practical considerations of costs and politics together with utility and fairness can help practitioners reach decisions about what can be done in practice, and what the opportunity costs might be. Taking into account each of these conditions can help ensure, for example, that a program cannot be justified based on its political feasibility alone (e.g., it is a "pet" issue of political leaders), but must also have utility in the particular local community of interest and be based on sound evidence. ${ }^{26}$ The balancing of multiple considerations recognizes that ethical principles and values can, at times, conflict.

\section{Assessing the utility and fairness of a given proposal provides a foundation for determining which public health actions might be ethically justified, or what should be done. Incorporating the more practical considerations of costs and politics together with utility and fairness can help practitioners reach decisions about what can be done in practice, and what the opportunity costs might be.}

vaccination with individuals' autonomy. Since public health seeks to do good in communities while doing minimal harm to individuals or groups, the principles of beneficence and non-maleficence are clearly relevant. Indeed, these bioethical principles are important ethical tenets for all health-related professions (including public health), even if they are not an integral part of the framework we advance here.

\section{Application of the Framework}

We do not consider each item in the framework independently morally binding; we recognize that there will be times when actions are approved despite one or more of the considerations not being met. We also recognize that there may not always be clear distinctions among the six considerations. For instance, cost assessment and political feasibility can be interrelated. The cost of a proposed program can have a direct impact on the political support for it, particularly if implementing the program would limit the ability to carry on existing programs. In that light, it is our intention that policymakers and practitioners use this framework as one tool to assist in their decisionmaking processes.

The elements of our framework are individually important, even if no single element is most important. Combined, their value is enhanced. Assessing the utility and fairness of a given proposal provides a foundation for determining which public health actions might be ethically justified, or what should be

\section{An Example: Planning for an Avian Influenza} Pandemic

Some public health practitioners have voiced concerns that excessive resources are being spent on emergency preparedness vis-à-vis the enormous amount of unmet need for other public health services in their communities. ${ }^{27}$ In anticipation of human-to-human transmission of avian influenza and a resulting pandemic, local public health officials must make decisions about the extent to which they will prepare for such an emergency. Many decisions must be made about the types of targeted, non-pharmaceutical interventions that will be implemented prior to the availability of a vaccine. Numerous potential ethical challenges associated with planning for and implementing these interventions exist, such as the following: anticipation of disproportionate impact of interventions on vulnerable populations, limitations on individual liberties to improve community health, and decisions about the allocation of current resources for planning and preparation in lieu of other public health activities. The application of the framework proposed above may assist in anticipating and mitigating such challenges, and may provide a guide for thorough ethical analysis.

Some non-pharmaceutical interventions that communities may plan to employ to prepare for and respond to avian influenza include the following: population surveillance; public education and community communication efforts; responder training; social distancing (e.g., travel restrictions, workplace distancing, 
home isolation/quarantine, school and childcare closings); and, practice drills and tabletop exercises. ${ }^{28}$ All of these interventions require significant resources to develop, test, and execute. Some would involve limitations on individuals' liberty (e.g., isolation or quarantine), and others are likely to have a disproportionate impact on the working poor and other vulnerable populations (e.g., school and child-care closings). The first consideration of our framework would require policymakers and practitioners to evaluate the utility and overall value that preparation for such interventions would bring to their community as a whole and to various stakeholder groups within the community. They would explicitly assess both the risk to their community of avian influenza and the goals of preparedness activities, and would determine whether various preparedness strategies would likely benefit the community. Decision-makers should consider the following questions: What is the value to this community of running practice drills in alternative care sites? How useful will it be to establish guidelines for school closure?

Second, policymakers would examine the evidence about the potential for avian influenza to spread to their community, and for whether preparedness activities are known to be effective. This consideration may be particularly difficult to meet in the context of emergency preparedness because experts must speculate about future community need and effectiveness of interventions on future threats. For instance, there may be solid evidence about the effectiveness of social distancing efforts during the SARS outbreak in recent years, but can experts expect individuals within communities to behave in the same way during an avian influenza outbreak?

Applying the consideration of fairness, policymakers would assess who would benefit from the planning for the pandemic. They would anticipate which sectors of society would likely bear the burdens associated with the proposed activities, assess how those burdens would compare to the expected benefits, and identify any probable disparate impact of interventions on particular populations to determine whether the balance of burdens and benefits would be acceptable or require modifications. For example, decision-makers might model the economic impact of closing daycare centers on the working poor in the community and also propose safeguards to offset the predicted disparate impact.

To ensure that the accountability consideration is met, policymakers would be required to clearly communicate the values- and evidence-based underpinnings of the planned interventions to the affected communities. For example, publicly announcing criteria to be used for determining a school closure and clearly stating reasons for choosing such criteria would create transparency in the proposed process and provide opportunities for community scrutiny. Public participation in emergency planning and preparedness exercises could also increase accountability. By "experiencing" actions such as the rationing of limited resources, community members can become educated about the reasons and values that underlie decisions made by public health workers. Participation may not only lead to accountability and greater understanding, but it may even lead to acceptance of difficult public health actions (e.g., rationing decisions), if the action is considered legitimate. ${ }^{29}$

The consideration of a cost assessment would require those designing such a program to predict both the monetary costs associated with planning and executing the proposed preparedness interventions and the tradeoffs associated with investing in such activities. Questions practitioners might consider include: What would it cost to run an effective community education program? Will it ultimately be more efficient to hold frequent tabletop exercises or to run actual drills? Should we hire another nurse to work on emergency preparedness or to work in the immunization clinic?

Finally, decision-makers would need to consider whether their plans and proposed interventions are feasible within their particular political environment. Are the community's elected officials aware of the rationale behind the proposed plan to provide care for certain individuals in alternative care sites with limited resources? Are they committed to supporting school closures and travel restrictions when necessary to help limit the spread of disease? Inevitably some conflicts will arise in the process of planning for a public health crisis, and decision-makers attempting to meet all the considerations of the framework will be required to synthesize their judgments to make final determinations. No framework will eliminate the need for judgment, but frameworks can guide policymakers and practitioners through a thorough examination of important ethical considerations.

\section{Discussion of the Framework}

We believe the proposed framework has several strengths. First, decision-makers can apply it in multiple ways. It can be used to assess areas of ethical tension in practice and to provide a structured way in which to deliberate and ultimately manage ethical issues when they occur. Applied prospectively by "upstream" decision-makers, these considerations may even help to avoid the unintentional creation of some ethical tensions for those who will ultimately execute and be affected by the programs. As govern- 
mental bodies make strategic determinations about the types of public health services they intend to fund and administer, they could assess, in advance, the potential that programs and services have for engendering ethical challenges. Prospective recognition of potential problem areas could go a long way toward reducing the overall level of ethical tension in public health practice, tension which we believe can at times inhibit effective public health service provision. If decisions at the policy level were able to reduce the likelihood that practitioners spend valuable time struggling with a challenging ethical issue (such as when a politician directs funds toward a program that is not a chief health concern in their community), practitioners may be better able to follow best practices and be more capable of delivering services efficiently.

Second, we balance several competing considerations rather than setting priorities among principled approaches to public health. The organizing principle of the Gostin and Powers model is the centrality of social justice to public health. ${ }^{30}$ For that reason, it will resonate with public health advocates and many scholars. A focus on justice very effectively highlights the need to make decisions that help disadvantaged populations. But attending to the concept of justice alone does not enable policymakers to, for example, make distinctions among and between disadvantaged populations in order to allocate scarce public health resources. Our concern is that social justice will become to public health ethics what autonomy has become to bioethics - a concept that overrides all other factors.

Third, we recognize that this framework will have little utility if practitioners and policymakers do not find it helpful. Proposals for ethical consideration in public health practice will not be successful if they are not sufficiently flexible, accessible, or acceptable for use in daily practice or in policymaking. Stated broadly, we believe that much more empirical research needs to occur in order to form a deeper understanding of public health ethics. While our empirical experiences provide the basis for the framework we advance here, our approach remains to be tested and evaluated by public health practitioners.

Fourth, we have focused our energies on public health ethics in the U.S. context, but we believe our proposed framework is flexible enough to be applied in other environments that are more centralized than the U.S. public health system. A more centralized role for government in the planning, administration, and delivery of public health services may render frameworks even more useful as explicit methods for assessing potential ethical challenges. For example, consideration of political feasibility may be more pressing in countries where public health systems are centralized, and where health ministries are responsible for the entirety of public health practice. In nations or communities with fewer resources, the framework may guide policymakers toward very different actions, depending on the utility, evidence, fairness, accountability, cost assessments, and determinations of political feasibility. Given that many issues central to public health require us to define communities more broadly than do political borders, it may be instructive to explore the usefulness of frameworks in a global context. Environmental health efforts to maintain air and water quality or infectious disease containment strategies often cannot be limited to individual countries but must be coordinated among many political bodies to be effective. Future work developing frameworks or other analytic approaches in public health ethics will likely develop within a more global context than has traditional bioethical inquiry in order to be applicable to broadly defined communities. ${ }^{31}$

There are likely to be various objections to the framework we have outlined here. First, as just discussed, public health practitioners and policymakers might argue that it is not sufficiently pragmatic to be useful in practice. This is why we stress that frameworks ought to be tested among practitioners to assess their potential utility and identify any barriers to their use. Following pilot tests, we would modify the proposed framework to be maximally effective for policymakers and practitioners.

Second, some may contend that our proposal does not go far enough in helping practitioners and policymakers engage in broader questions about which of multiple competing public health values ought to receive priority in delineating the scope and emphasis of the field. One's conviction, for example, that social justice is the pre-eminent public health value will define his or her broad conception of the boundaries of public health..$^{32}$ Others, however, might caution against such a broad view of public health, believing instead that population protection, from communicable diseases or other threats, is the most important public health value. Clearly these are important questions for the field of public health to address, and we expect that some macro-level political deliberation about values and principles would precede the application of our framework. However, we feel that the balancing of principled concerns with practical demands required by our framework could help to structure such deliberations. In fact, because the debate over which principles ought to take priority in public health is likely to be contentious and protracted, Daniels and Sabin's emphasis on the fair and legitimate process for making such deliberations has 
merit. ${ }^{33}$ In order to apply a process-based approach to decision-making, however, one must be comfortable with the possibility that similar situations may result in very different outcomes because of differences among the processes each employed. The Daniels and Sabin approach makes sense as an interim strategy, but begs the question of just how practitioners are to make the determinations without a framework that recognizes the need to balance the competing principles.

\section{Conclusion}

Ethical issues in public health can have a significant impact on the health of communities if they impede efficiencies and best practices. Competing needs for resources and a plurality of values can challenge public health policymakers and practitioners to make fair and effective decisions for their communities. We have proposed a framework built upon a set of pragmatic considerations that has the potential to illuminate possible ethical tensions and create the opportunity to resolve them in day-to-day public health practice. Such a framework is an attempt to connect public health ethics more closely with public health policy, an objective that Ezekiel Emanuel has characterized as asking ethicists to reach beyond the abstract and be "grounded in the real world" and at the same time asking policymakers to examine their underlying values so that decisions are both legitimate and practical. ${ }^{34}$ We hope to advance the dialogue on public health ethics to consider pragmatic ways both to resolve ethical issues when they emerge (secondary prevention), or, as we have suggested, before they even surface (primary prevention).

\section{Acknowledgements}

This work was supported in part by grants from the Greenwall Foundation and from the Robert Wood Johnson Foundation, Health Care Financing and Organization Initiative.

\section{References}

1. Some of the recent work that makes this point include the following: R. Bayer, L. Gostin, B. Jennings, and B. Steinbock, eds., Public Health Ethics: Theory, Policy and Practice (New York: Oxford University Press, 2007); R. Bayer and A. Fairchild, "The Genesis of Public Health Ethics," Bioethics 18, no. 6 (2004): 47392; J. F. Childress, R. R. Faden, R. D. Gaare, L. O. Gostin, J. Kahn, R. J. Bonnie, N. E. Kass, A. C. Mastroianni, J. D. Moreno, and P. Nieburg, "Public Health Ethics: Mapping the Terrain," Journal of Law, Medicine छ Ethics 30, no. 2 (2002): 170-78; D. Callahan and B. Jennings "Ethics and Public Health: Forging a Strong Relationship," American Journal of Public Health 92, no. 2 (2002): 169-76; and N. Kass, "An Ethics Framework for Public Health," American Journal of Public Health 91, no. 11 (2001): 1776-82.

2. Id. (Kass, 2001); N. Kass, "Public Health Ethics: From Foundations and Frameworks to Justice and Global Public Health," Journal of Law, Medicine छ Ethics 32, no. 2 (2004): 232-42; and J. Thomas, M. Sage, J. Dillenberg, and V. J. Guillory, "A Code of Ethics for Public Health," American Journal of Public Health 92, no. 7 (2002): 1057-59.

3. Greenwall Foundation Pilot Grant, "Public Health Ethics in Practice: A Pilot Study of Day-to-Day Ethical Issues in Public Health Practice in Michigan," funded from 2006-2008.

4. See, for example, Kass (2001), supra note 1.

5. For example, L. Gostin and M. Powers, "What Does Social Justice Require for the Public's Health? Public Health Ethics and Policy Imperatives," Health Affairs 25, no. 4 (2006): 1053-60.

6. See Bayer and Fairchild (2004), supra note 1, among others.

7. C. Schneider, The Practice of Autonomy: Patients, Doctors, and Medical Decisions (New York: Oxford University Press, 1998).

8. R. G. Bernheim, "Public Health Ethics: The Voice of Practitioners," Journal of Law, Medicine $\&$ Ethics 31, no. 4, Supplement (2003): 104-9; see Bayer and Fairchild (2004), supra note 1; Childress et al., supra note 1; Callahan and Jennings, supra note 1; and Kass (2004), supra note 2, among others.

9. See both M. Wynia, "Oversimplifications I: Physicians Don't Do Public Health," American Journal of Bioethics 5, no. 4 (2005): 4-5; and M. Wynia, "Oversimplifications II: Public Health Ethics Ignores Individual Rights," American Journal of Bioethics 5, no. 5 (2005): $6-8$

10. See Gostin and Powers, supra note 5; Bayer and Fairchild, supra note 1; P. Nieburg, R. G. Bernheim, and R. Bonnie, "Ethics and the Practice of Public Health," in R. Goodman, et al., eds., Law in Public Health Practice (New York: Oxford University Press, 2003); Childress et al., supra note 1; Callahan and Jennings, supra note 1; Kass (2001), supra note 1.

11. See Kass (2001), supra note 1; Childress et al., supra note 1; and Nieburg et al., supra note 10.

12. See Kass (2001), supra note 1.

13. See Childress et al., supra note 1.

14. See Nieburg et al., supra note 10.

15. See Gostin and Powers, supra note 5.

16. See N. Daniels, "Justice, Health and Healthcare," American Journal of Bioethics 1, no. 2 (2001): 2-16; or N. Daniels and J. E. Sabin, "Limits to Healthcare: Fair Procedures, Democratic Deliberation, and the Legitimacy Problem for Insurers," Philosophy and Public Affairs 26, no. 4 (1997): 303-50.

17. See, for example, J. F. Childress and R. G. Bernheim, "Beyond the Liberal and Communitarian Impasse: A Framework and Vision for Public Health," Florida Law Review 55, no. 5 (2003): 1191-1219.

18. Institute of Medicine, The Future of the Public's Health in the 21st Century (Washington, D.C.: National Academy Press, 2003).

19. See Thomas et al., supra note 2.

20. T. Beauchamp and J. Childress, Principles of Biomedical Ethics (New York: Oxford University Press, 1989); M. Maciosek, A. Coffield, N. Edwards, T. Fottemesch, M. Goodman, and L. Solberg, "Priorities Among Effective Clinical Preventive Services," American Journal of Preventive Medicine 31, no. 1 (2006): $52-61$.

21. C. Keane, J. Marx, and E. Ricci, "Local Health Departments' Mission to the Uninsured," Journal of Public Health Policy 24, no. 2 (2003): 130-49.

22. R. Epstein, "Let the Shoemaker Stick to His Last: A Defense of the 'Old' Public Health," Perspectives in Biology and Medicine 4, no. 3, Supplement (2003): S138-59; M. Hall, "The Scope and Limits of Public Health Law," Perspectives in Biology and Medicine 46, no. 3, Supplement (2003): S199-S209.

23. L. O. Gostin and M. G. Bloche, "The Politics of Public Health," Perspectives in Biology and Medicine 46, no. 3 (2003): S16075 ; id. (Epstein).

24. F. Mullan, "Don Quixote, Machiavelli, and Robin Hood: Public Health Practice, Past and Present," American Journal of Public Health 90, no. 5 (2000): 702

25. L. Block, "Antifluoridationists Persist: The Constitutional Basis for Fluoridation," Journal of Public Health Dentistry 46, no. 4 (1986): 188-98; Centers for Disease Control and Prevention, National Center for Chronic Disease Prevention and Health Promotion, Division of Oral Health, "Achievements in Public 
Health, 1900-99: Fluoridation of Drinking Water to Prevent Dental Caries," Morbidity and Mortality Weekly Report 48, no. 41 (1999): 933-40.

26. M. Wynia, "Risk and Trust in Public Health: A Cautionary Tale," American Journal of Bioethics 6, no. 2 (2006): 3-6.

27. J. Hyde, K. Basil, L. Martinez, M. Clark, and K. Hacker, "Better Prepared but Spread Too Thin: The Impact of Emergency Preparedness Funding on Local Public Health," Disaster Management Response 4, no. 4 (2006): 106-13.

28. U.S. Department of Health and Human Services, Centers for Disease Control and Prevention, Interim Pre-pandemic Planning Guidance: Community Strategy for Pandemic Influenza Mitigation in the United States, 2007, available at <http:// www.pandemicflu.gov/plan/community/community_mitigation.pdf> (last visited August 16, 2007).
29. A. Gutmann and D. Thompson, "Deliberating about Bioethics," Hastings Center Report 27, no. 3 (1997): 38-41.

30. See Gostin and Powers, supra note 5; see also M. Powers and R. Faden, Social Justice: The Moral Foundations of Public Health and Health Policy (New York: Oxford University Press, 2006).

31. See Kass, supra note 2.

32. See Gostin and Powers, supra note 5.

33. See Daniels and Sabin, supra note 16.

34. E. Emanuel, "Foreward," in M. Danis, C. Clancy, and L. Churchill, eds., Ethical Dimensions of Health Policy (New York: Oxford University Press, 2002): at x. 